\title{
IMPACT PARAMETER DEPENDENT CHARGE EXCHANGE STUDIES WITH CHANNELED HEAVY IONS
}

\author{
D. DAUVERGNE, M. CHEVALLIER, J.-C. POIZAT, C. RAY, E. TESTA \\ Institut de Physique Nucléaire de Lyon, CNRS- IN2P3 and Université Claude Bernard \\ Lyon 1, F-69622 Villeurbanne, France
}

A. BRÄUNING-DEMIAN, F. BOSCH, S. HAGMANN, C. KOZUHAROV, D. LIESEN, P. MOKLER, TH. STÖHLKER, M. TARISIEN ${ }^{\dagger}$, P. VERMA

Gesellschaft für Schwerionen Forschung (GSI), D-64291 Darmstadt, Germany

C. COHEN, A. L'HOIR, J.-P. ROZET, D. VERNHET

Institut des Nano-Sciences de Paris, CNRS-UMR75-88, Universités Paris VI et Paris VII, 75251 Paris cedex 05, France

H. BRÄUNING

Institut für Atom- und Molekülphysik, Justus Liebig Universität, D-35392 Giessen, Germany

\section{TOULEMONDE}

Centre Interdisciplinaire de Recherche Ions-Lasers, UMR 11 CEA-CNRS, 14040 Caen cedex, France

\begin{abstract}
We use decelerated (below $20 \mathrm{MeV} / \mathrm{u}$ ) H-like heavy ions extracted from the GSI-ESR storage ring to study electron capture processes such as Radiative Electron Capture (REC) and Mechanical Electron Capture (MEC) in channeling conditions. With the help of simulations, we show that MEC occurs at relatively large impact parameters into highly excited states. REC studies provide information about the electron gas polarization.
\end{abstract}

\footnotetext{
${ }^{\dagger}$ Present address: CENBG, CNRS-IN2P3 and Université Bordeaux 1, Le Haut Vigneau, 33175 Gradignan cedex, France.
} 


\section{Introduction}

Bare heavy ions can be decelerated down to a few $\mathrm{MeV} / \mathrm{u}$ at the Experimental Storage Ring facility (GSI, Germany). Such ions are very far from their charge equilibrium in matter, since their velocity is much smaller than the velocity of their inner-shell bound electrons. The extraction of such beams allows one to study their collision with solid targets. Their interaction with matter should provide a very strong perturbation, along with a very high cross section for electron capture.

We present here some results concerning the impact parameter dependence of the electron capture processes competing inside a crystalline target under alignment conditions.

Indeed, channeling of charged particles in a crystal leads to a redistribution of the ion flux inside the target. Channeled ions have a restricted accessible transverse space, which is determined by their transverse energy [1]. In the continuum potential approximation, the collisions of particles along atomic strings or planes are described as deflections by a continuum potential, which is averaged along the crystallographic direction. With the help of simulations, detailed information can be extracted on the impact parameter dependence of interaction processes such as charge exchange and energy loss, provided that the transverse energy of channeled ions can be determined. This is what has been done in this work, which presents part of the results obtained recently with decelerated heavy ions at $20 \mathrm{MeV} / \mathrm{u}$ and below.

\section{Experiment}

A detailed description of the production of decelerated H-like heavy ions has been given in ref. [2].

Briefly, some $10^{7}$ highly-charged ions, accelerated up to a few hundreds of $\mathrm{MeV} / \mathrm{u}$ by the heavy-ion synchrotron SIS-18, are fast extracted. They are further totally stripped in a thick stripper foil placed between the SIS and the storage ring ESR, and then injected at once inside the ESR. After cooling and deceleration, they are extracted continuously from the ring by means of radiative recombination inside the electron cooler, which changes the magnetic rigidity relative to stored bare ions. Due to different orbits, the H-like ions can be deflected into the extraction channel by a septum magnet. The electron current in the cooler is tuned in order to adjust the extracted ion beam intensity. Thus, within a full cycle of about 6 minutes, a continuous beam is extracted during 4 minutes, exponentially decreasing with time from a few $10^{4}$ ions $/ \mathrm{s}$ 
down to $5.10^{3}$ ions/s typically. The beam is transported towards the experimental cave and focused with the most possible parallel optics on the crystal target. Behind the target, transmitted ions are analyzed in charge and momentum by a magnetic spectrometer. As targets, we have used silicon crystals of various thicknesses and orientations. Before setting a crystal under vacuum, its surfaces were cleaned by means of a fluorhydric acid solution.

In all experiments, X-rays are detected at $90^{\circ}$ from the beam direction. The target is biased, in such a way that emitted electrons from the surfaces are collected by grounded silicon detectors located in front of the two surfaces of the thin crystal.

At the focal point of the spectrometer, a position sensitive detector (microchannel plate counter with delay-line readout) allows to detect the selected ions and to perform an event by event acquisition containing coincidences between X-rays, electron multiplicities and transmitted ions of identified magnetic rigidity.

\section{Results and simulations}

We will concentrate mainly on the experiment performed with $20 \mathrm{MeV} / \mathrm{u} \mathrm{U}^{91+}$ ions channeled in an 11.7 micrometer thick crystal. Some of the results have been presented in ref. [3]. Here we add more refined analysis obtained by means of simulations, which need to be described first.

\subsection{Principles of the simulations}

These simulations are aimed to estimate the mean number of electron captures by aligned projectiles as a function of their transverse energy, assuming statistical flux equilibrium for channeled ions. The simple idea behind the statistical flux equilibrium is that channeled ions of given transverse energy explore uniformly all their accessible transverse space. Thus the transverse energy of a channeled ion defines its impact parameter distribution along the path inside the crystal. The charge state at emergence is then calculated by injecting in Monte Carlo simulations an impact parameter dependent capture probability. Such simulations have the advantage to avoid full trajectory calculations, which spares computation time (trajectory calculations are made once for each transverse energy in order to implement the impact parameter distributions). The drawback is that multiple charge exchange effects, arising when an ion approaches close to a string or a plane, cannot be taken into account. This is why we have limited these simulations to ions with a relatively low transverse energy, i.e. for which each single charge exchange event can be considered independently. For high transverse energy ions, suffering close collisions along atomic strings, full Monte Carlo calculations are needed to 
follow the evolution of the electronic configuration. Such a work, devoted to the study of a "super-density effect" associated to the very high rate of atomic collisions near strings, has been described separately [4].

The transverse energy distribution of the incoming beam depends on the crystal orientation and on the beam angular distribution. The latter is a parameter in the simulations, which has to be adjusted according to our observations. We take into account the dechanneling effects that tend to increase the transverse energy of channeled ions inside this relatively thick crystal. The number of electron captures is estimated as following:

REC probabilities are proportional to the sampled local electron density, and depend on the occupation of inner shells (REC cross sections decrease as the mean quantum number $\mathrm{n}$ of the final state increases). MEC probabilities are adjusted as a function of impact parameter to get the best possible agreement with the various measurements. A small probability of MEC into the thin amorphous surface layers is also considered, which does not depend on the ion transverse energy.

\subsection{Results}

Figure 1 shows the charge state distributions obtained for random, (110) planar and $<110>$ axial orientations, after crossing a $11.7 \mu \mathrm{m}$ thick crystal. In such a thick crystal, the charge equilibrium is reached only for a random target orientation. In channeling conditions, the charge state distributions are much broader and extend from frozen $91+$ ions to very low charge states.

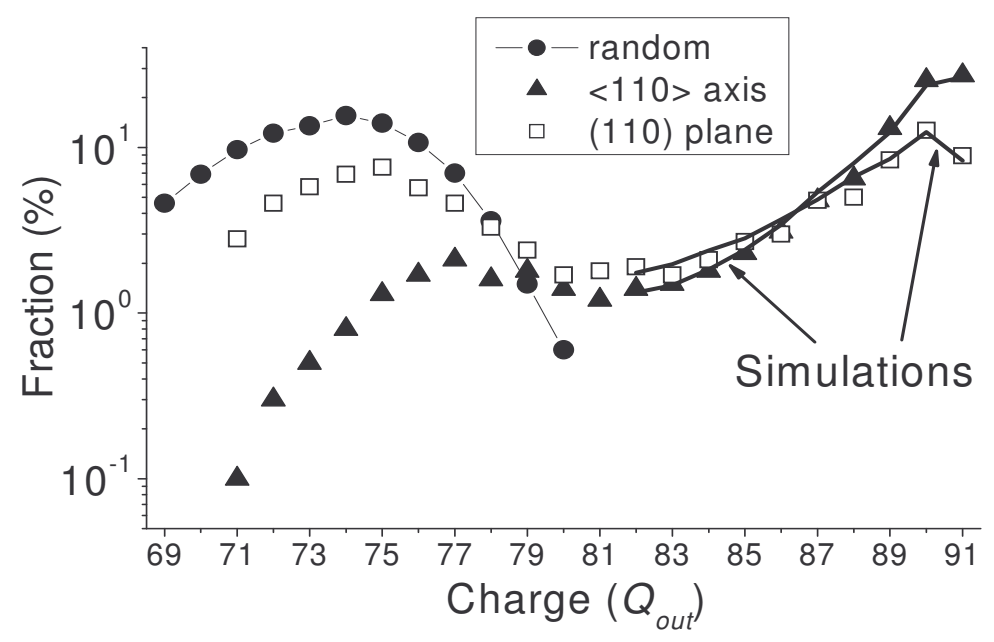

Figure 1. Charge state distributions obtained for $20 \mathrm{MeV} / \mathrm{u}$ incident $\mathrm{U}^{91+}$ ions on a $11.7 \mu \mathrm{m}$ thick Si crystal for various orientations. The thick solid lines are simulations (see text). 
These distributions reflect the transverse energy distributions of channeled ions, frozen ions being the best channeled ones. The results of the simulations are superimposed. They agree fairly well, both in the shape and in the absolute amplitude of the distributions (although ions with a high transverse energy are not considered in the simulations, we evaluated this fraction of ions that reach charge states below $82+$ ).

Tilting the crystal by an angle $\delta \psi$ relative to the incident beam increases the transverse energy $\mathrm{E}_{\perp}$ of each incident particle by the amount $\delta \mathrm{E}_{\perp}=\mathrm{E}(\delta \psi)^{2}$, where $\mathrm{E}$ is the total ion kinetic energy. Thus the charge state distribution strongly depends on the crystal orientation. This is shown in figure 2, where we present the evolution of charge fractions 91 and 87 as a function of the tilt angle relative to the $\langle 110\rangle$ axis. The frozen ion fraction disappears almost completely at a tilt angle which is less than half the channeling critical angle. The fraction of the lower charge state 87 , increases for increasing small angles and rapidly decreases at angles only slightly larger than for frozen ions. This shows that many electron capture events occur for ions with relatively small transverse energy, since an incidence angle of $0.04^{\circ}$ corresponds to a minimum distance of approach to atomic strings of about $0.4 \AA$ for ions entering the crystal at the center of the channel. Again the simulations are able to reproduce the data.

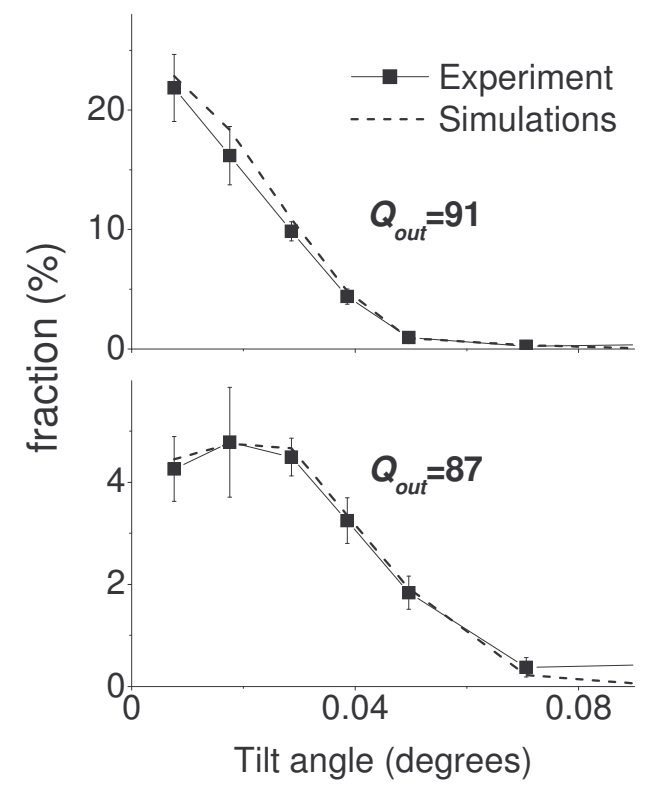

Figure 2. Evolution, with the angle relative to the $<110>$ axis, of charge state fractions 91 and 87 for $20 \mathrm{MeV} / \mathrm{u}$ incident $\mathrm{U}^{91+}$ on a $11.7 \mu \mathrm{m}$ thick Si crystal. The dashed lines are simulations. 
Information on the nature of electron capture is deduced from the X-ray observation. This is illustrated in figure 3, where two X-ray spectra are shown: one for axial crystal orientation (in coincidence with $90+$ transmitted ions), and the other one for a random orientation. Both spectra show mainly transitions into $n=1$ and $n=2$ levels of the uranium projectiles. They correspond either to inner-shell transitions (K and L-lines) or to REC into K- and L-shells. As MEC occurs into excited states, the filling of the $\mathrm{K}$ and L-shells following MEC causes the emission of $\mathrm{K}$ and $\mathrm{L}$ lines. In the random spectrum, a large fraction of the L-lines is also due to the creation of $\mathrm{L}$ vacancies once the charge equilibrium is reached in this thick target, or at least once the L-shell is filled. Note that Kand L-REC lines are almost absent from the random orientation spectrum. The reason is that those inner shells are very rapidly filled inside the target by MEC, which prevents REC from occurring. On the contrary, ions emerging as $90+$ under axial alignment are well channeled ions, for which MEC is strongly reduced inside the crystal.

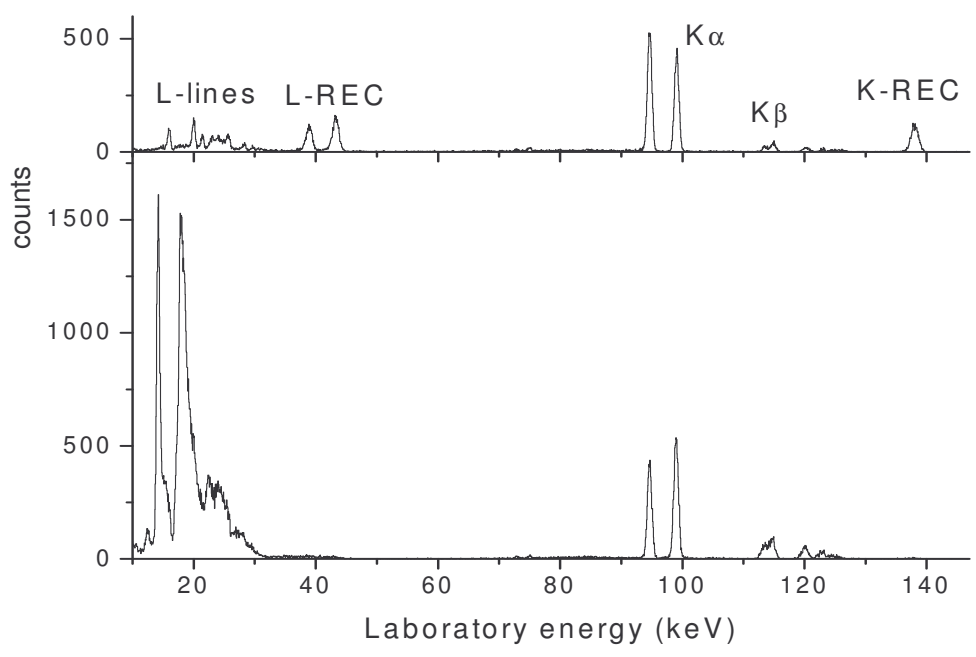

Figure 3. X-ray spectra recorded at $90^{\circ}$ for $20 \mathrm{MeV} / \mathrm{u}$ incident $\mathrm{U}^{91+}$ on a $11.7 \mu \mathrm{m}$ thick Si crystal. Upper spectrum: axial orientation, in coincidence with $\mathrm{U}^{90+}$ transmitted ions. Lower spectrum: random incidence. The spectra are normalized to the same number of transmitted ions.

For those ions, REC is the dominant capture process, and the corresponding peaks are easily observed. Here, even $\mathrm{K}_{\alpha}$ lines are mainly due to the decay of electrons captured by REC into low j-values of the L-shell (note that the ratio $\mathrm{K}_{\alpha 1} / \mathrm{K}_{\alpha 2}$ is inverted on the two spectra in fig. 3). Thus, REC and MEC capture rates can be evaluated for any transverse energy of channeled ions, that are 
selected either by their charge state at emergence, or by their electron multiplicities from the crystal surface. This is shown in figure 4, where average MEC and REC rates are represented as a function of the minimum distance of approach to the atomic strings along the path inside the crystal (axial orientation). This curve is obtained as a direct output of the simulations. One can see the very fast increase of MEC events when ions are able to approach the strings at distances smaller than $0.4 \AA$ to atomic strings. REC contributes significantly at large distances. However, no increase of the REC yield is found close to the target atoms, although the mean electron density sampled by ions with increasing transverse energy increases. This is again due to the rapid filling of inner shells by MEC and electron cascades close to the entrance of the crystal (ions with parallel incidence with the crystal axis penetrate the crystal at the minimum distance of approach to strings, and so the MEC rate is maximum there).

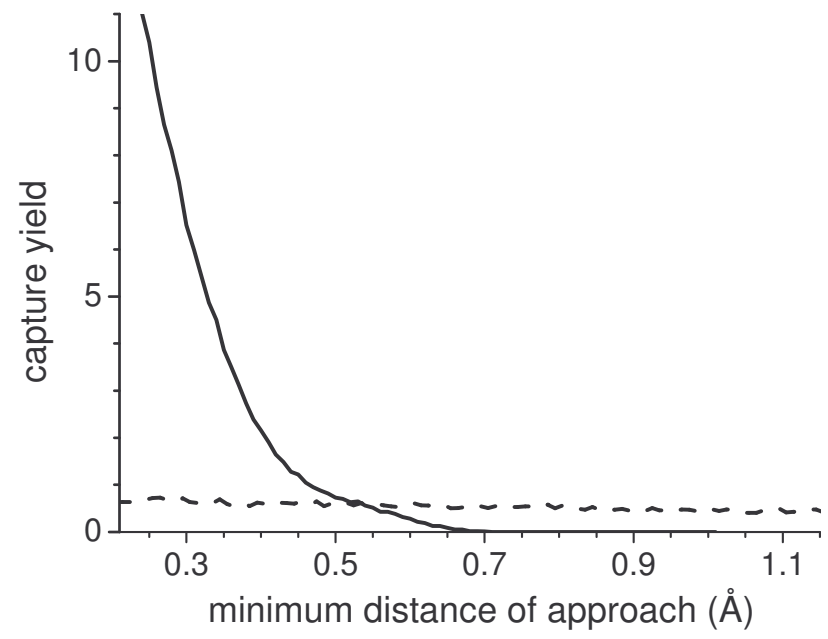

Figure 4. Estimates, deduced from simulations, of the total capture yields for $20 \mathrm{MeV} / \mathrm{u}$ incident $\mathrm{U}^{91+}$ inside the $11.7 \mu \mathrm{m}$ thick Si crystal, as a function of the minimum distance of approach to the $<110>$ atomic strings for channeled ions. Solid line: MEC yield, dashed line: REC.

\section{Discussions}

\subsection{Mechanical Electron Capture}

The knowledge of the complete impact parameter distribution for channeled ion trajectories inside the crystal is needed to extract the atomic impact parameter 
probabilities of MEC. In particular, one has to take into account the thermal vibrations of the target atoms, and dechanneling effects. This makes the dependence with atomic impact parameter of MEC significantly narrower than shown in Fig.4. Our experiments are mainly relevant in the impact parameter region $0.2-0.4 \AA$, where a maximum of constraints on the fitting procedure can be extracted from the experimental observations. Actually, we show in Fig. 4 an effective capture probability, i.e. the probability to capture an electron and not to lose it by ionization afterwards. At distances of approach smaller than $0.2 \AA$, a much thinner crystal would be needed to limit the number of electron captures so that information can still be extracted from X-rays (no more L-lines consecutive to capture are emitted beyond 9 captured electrons). At distances of approach larger than $0.4 \AA$, the origin of the uncertainties is the determination of the absolute REC probabilities, of the MEC capture yield in the thin surface amorphous layers and the knowledge of the beam angular divergence.

The impact parameter dependent MEC probabilities have been deduced from the capture yield presented in Fig.4, and the impact parameter distributions calculated by means of trajectory simulations. They have been compared with CDW-EIS calculations performed by P. Abufager et al. [5]. These calculations were limited up to $n=5$ due to the very large complexity of such analytical calculations for higher projectile n-shell values. These calculations show that for $\mathrm{n}<6$ MEC occur mostly at impact parameters smaller than $0.25 \AA$, i.e. in a region where our experiments do not provide very accurate information. In fact, MEC capture into these "inner" shells is not the dominant process.. Actually, integrated CDW calculations show that the cross section is maximum for final states equal to 5 and 6 , and only slowly decreases for higher n-shells. Additional CTMC calculations of MEC probabilities as a function of impact parameter are now in progress.

\subsection{Radiative Electron Capture}

The analysis of the K- and L-REC peaks (energy values, shape and amplitude) provide detailed information on the ion-electron interaction by such highly charge ions in a dense electronic medium. We have reported in ref. [6] that a shift of about $-100 \mathrm{eV}$ of the REC lines is observed compared to the calculated values corresponding to the capture of a free electron isolated in vacuum. This shift is attributed to the dynamic electron gas polarization by the high projectile charge, slowly moving inside the medium. This energy shift is found in agreement with values calculated by the linear response theory [7]. 
Another consequence of the dynamic response of the electron gas is the local increase of the local electron density at the ion site, which may increase the REC yield compared to a non perturbed electron gas. Indeed, we do observe an increase of about $40 \%$ [6]. This increase of the local electron density is much smaller than predicted by the linear response theory. Actually, this is not surprising, because the first order perturbation calculations are certainly not valid for such a system. Non perturbative calculations are obviously needed to provide an accurate description of the electron gas polarization by such high charges moving in matter at these velocities.

\section{Conclusion}

We have shown that charge exchange by decelerated highly charged ions can be studied in details as a function of impact parameter using crystal channeling. For $20 \mathrm{MeV} / \mathrm{u} \mathrm{U}^{91+}$ ions, non radiative capture (MEC) into highly excited states is the dominant capture process at small impact parameters. Complete calculations of the impact parameter dependence of MEC are still in progress, the analytical CDW-EIS being limited to relatively low-lying states. REC is the dominant capture mechanism at large distances from the target atoms, and provides interesting information on the dynamic electron gas polarization. In a more general review on these experiments, to be published, we will also discuss particular aspects of energy loss, the very high charge of channeled ions being responsible for very high energy loss rates.

\section{Acknowledgments}

We gratefully thank P. Abufager and R. Rivarola who provided us with calculations of electron capture.

This work was supported by the French - German GSI-IN2P3 collaboration agreement 97-35.

\section{References}

1. D. S. Gemmell, Rev. Mod. Phys. 46 (1974) 129.

2. H. T. Prinz et al., Hyperfine Interactions Cf22, 1729 (1995).

3. D. Dauvergne et al., Nucl. Instr. And Meth. Phys. Res. B205, 773 (2003).

4. A. L'Hoir et al., to be published in Nucl. Instr. and Meth. Phys. Res. B.

5. P. Abufager and R. Rivarola, private communication

6. E. Testa et al., to be published in Nucl. Instr. and Meth. Phys. Res. B

7. P. M. Echenique, R. H. Ritchie, and W. Brandt, Phys. Rev. B20, 2567 (1979). 